\title{
LEVELS OF LYMPHOCYTE SUBPOPULATIONS IN PERIPHERAL BLOOD AMONG PATIENTS WITH DIABETES
}

\author{
S Ganeva ${ }^{1,3}$, K. Todorova ${ }^{1,3}$, Ts. Lukanov ${ }^{2,3}$, G. Rayanova ${ }^{1,3}$, S. Blajeva ${ }^{2,3}$ \\ ${ }^{1}$ Clinic of Endocrinology and Metabolic Diseases, UMHAT "Dr. G. Stranski" - Pleven, Bulgaria \\ ${ }^{2}$ Diagnostic Laboratory of Immunology, UMHAT "Dr. G. Stranski" - Pleven, Bulgaria \\ ${ }^{3}$ Medical University - Pleven, Bulgaria
}

\begin{abstract}
The aim of the study was to investigate the lymphocyte (lymph) subpopulations in peripheral blood as a part of the immune response among patients with diabetes mellitus type 1 (DMT1) and diabetes mellitus type 2 (DMT2). Patients and methods: $A$ prospective, cross-sectional, comparative, "case-control" study was conducted among 22 patients with DMT1 and 70 patients with DMT2. The levels of lymph subtypes [general nonspecific T-lymph (CD3+); T-helper lymph (CD4+); T-cytotoxic lymph (CD8+), natural killers [NK cells (CD3। CD16+/CD56)] and B-lymph (CD19+)] in blood was measured and compared by flow-cytometric analisys (FAC Sort, $B D$ ). Results were compared to those of 21 healthy persons. The data was processed using the statistics software. Results: Patients with DMT1 had longer duration of the disease, compared to patients with DMT2. No significant differences between arterial blood pressure, $\mathrm{H} 8 \mathrm{~A} 1 \mathrm{c}$ levels and lipid profile among the patients with DMT1 and DMT2 were present. There were no differences in the total leukocyte count between the groups (DMT1-6,91 \pm 1,32.10\%/l; DMT2-7,28 \pm $1,85.10^{9} /$; controls-6,89 $\left.\pm 1,07.10^{\circ} / \mathrm{l}\right)$. The results from the flowcytometric investigation showed significantly higher absolute number of T-all lymph (CD3+), Th lymph (CD4+) and all NK (CD3। CD16+/CD56), as well as a lower absolute number of Ts (CD8+) and $B(C D 19+)$ lymph among the diabetic patients compared to healthy subjects. The Th/Ts ratio in patients with DMT1 $(2,02 \pm 0,44)$ and DMT2 $(2,36 \pm 0,37)$ was also significantly higher compared to ratio of controls $(1,02 \pm 0,06)$. No significant differences were noted in the lymph subpopulations between the two groups with DM. Conclusions: Changes of lymph types in peripheral blood in diabetic patients demonstrate immune activation and dysregulation among the two types of diabetes.
\end{abstract}

Key words: type 1 diabetes mellitus, type 2 diabetes mellitus, flow cytometry, lymphocytes

Corresponding author: Silviya Ganeva, Clinic of Endocrinology and Metabolic Diseases, UMHAT "Dr. G. Stranski", Pleven, 91 "Vladimir Vazov" Street, Bg - 5800 Pleven, E-mail: doctorganeva@gmail.com, tel.: +3590896678598

ACCEPTED: 16 June 2020 


\section{INTRODUCTION}

$\mathrm{D}$ iabetes is defined as a syndrome of hyperglycemia due to absolute insulin deficiency and/ or loss of insulin effect. The classic diabetes mellitus type 1 (DMT1), according to Koch's postulates [1], is an organo-specific, autoimmune process characterized by $\beta$-cellular destruction and absolute insulin deficiency [2]. During the last 30 years, diabetes mellitus type 2 (DMT2) has been regarded to as a disease with complex pathogenesis. More recently, the disease was further referred to as a low-grade chronic inflammatory condition. Certain elements of $\beta$-cellular impairment in DMT2 overlap with those of DMT1 [3].

Among the patients with visceral obesity and DMT2, unlike DMT1, the identification of a specific antigen is difficult, because numerous organs (liver, adipose tissue, muscles, pancreas, vessels and hypothalamus) are involved in the pathogenetic process. Low-grade chronic inflammation, tissue destruction [4], the presence of extracellular-matrix products and cytokines from cellular apoptosis [5] in the circulation, are the cause for auto-antigens presentation and activation of T-reactive lymphocytes (lymph). So far, in experimental studies circulating damaging subclasses of antibodies (IgG) [6] have been described against GAD65 [7], IA2 and proinsulin, as well as active Tcellular response in patients with DMT2 [8].

The activation of adaptive immunity in obesity has many effects on the progression of insulin resistance (IR) and $\beta$ - cellular function. In experimental studies a correlation between the change in T-lymph populations and IR has been observed among animals $[9,10]$ and humans $[11,12,13]$. The expression of inflammatory cytokines (TNF- $\alpha, \mathrm{II}-1 \beta$ ) from cellular elements of adaptive immunity [14] has been acknowledged as the primary one. In 2001, Wilkin [15] first prompted the hypothesis that obesity and body mass index (BMI) are critical for the development of $\beta$-cells apoptosis and, that they are a so-called pathogenetic "bridge" between diabetes type 1 and 2 . According to this hypothesis, autoimmune-mediated $\beta$-cellular destruction is found in both types of diabetes, but the rate and speed of development is much lower and slower in DMT2.

The study of immune changes over the course of DMT2 could contribute to the better understanding of the pathogenetic mechanisms of the disease, as well as the selection of patients who would benefit from immune-modulating therapies in the clinical practice [16].

The aim of the study was to investigate the levels of lymph subpopulations in the peripheral blood as parts of the immune response: general nonspecific T-lymph (CD3+); T-helper lymph (CD4+); T-cytotoxic lymph (CD8+), natural killers [NK cells (CD3) CD16+/ CD56)] and B-lymph (CD19+) among patients with DMT1 and DMT2.

\section{PATIENTS AND METHODS}

A prospective, cross-sectional, comparative, "casecontrol" study was conducted among 22 (10 men and 12 women) patients with DMT1 and 70 patients (35 men and 35 women) with DMT2. The participants in the study were selected from hospitalized patients at the Endocrinology Clinic at University Hospital "Dr. G. Stranski", Pleven, Bulgaria after informed consent was singed. The results were compared to those of 21 healthy persons ( 9 men and 12 women) who were used as a control group. The diagnosis and type of diabetes were derived from the primary medical documentation and diagnostic criteria of the WHO were used in all cases [17]. Height and weight were measured and BMI was calculated for every patient. Their waist measurements were taken by standard methods. Arterial blood pressure was measured according to the recommendations of Thomas [18].

Blood samples were taken at fasting in the morning via venipuncture. The lab tests were provided in the clinical laboratory of the University Hospital, the activity of which is regulated by the National System for Laboratory Control. The plasma triglycerides, total cholesterol and HDL-cholesterol levels were examined via an enzyme-colorimetric method (automatic analyzer BA 400; BioSystems S.A, Spain). The values of LDL cholesterol were calculated by Friedewald's formula. The blood glucose levels were measured by hexokinase-enzyme method (GA 3, KABE Labortechnik, Denmark).

The general and differential leukocyte counts were conducted by an automatic hematologic analyzer (Micros 60 CS/CT-16; BX Horiba Diagnostics, France) for EDTA-venous blood. The samples were made according to the requirements of ICSH (International Committee for Standardization in Hematology). The lymph from same venous blood was studied within 2 hours of extraction via flow cytometry (FACS Calibur cytometer; Becton Dickinson, Heidelberg, Germany). The blood cells were processed with a combination of two monoclonal antibodies marked with two different fluorochromes. After erythrocyte lysis and double cleansing, the leucocytes connected with monoclonal antibodies were resuspended and fixed. By determining the fluorescence according to the bound monoclonal antibodies, the following cellular subpopulations were identified: CD3+ (T-all lymph), CD4+ 
(T- helper lymph [Th cells]), CD8+ (cytotoxic T lymph [Tc cells]), CD3-/CD16+56+ (NK cells) и CD19+ (Blymph) (Exbio, Praha, Czech Republic).

\section{Statistical analysis}

The results were analyzed as percentages using the computer software Cell Quest. The following formula was used to calculate the absolute value of lymphocyte fraction (\% lymphocyte type $X$ total lymph numbers $X$ 10 , where the information about the total lymph numbers was received from an automatic blood analysis). The data was processed using the statistics software STATGRAPHICS Centurion XVI.II. One-factor dispersion analysis (NOVA), parametric (T- test for two independent values) and nonparametric (KolmogorovSmirnov and Mann- Withey) tests were used for checking/verifying the hypotheses. The results were described with their average value, standard deviation, marked as "average \pm SD" and 95\% Cl (confidential interval). The deviations of empirical level of significance $\mathrm{P}<0.05$ were accepted as statistically significant.

\section{RESULTS}

The clinical characteristics of the diabetic patients and the control group are shown in Table 1.
The age of the participants with DMT2 (56,58 $\pm 2,25$ years) was statistically higher than the average age of those with DMT1 $(41,23 \pm 5,42$ years $)$ and the control group $(31,14 \pm 6,92$ years). The duration of the disease among patients with DMT1 (9,79 \pm 3,56 years) was longer than the duration of DMT2 $(6,12 \pm 1,45 ; p=0,02)$. No significant differences of arterial blood pressure, glycemic control (HBA1c) and lipid profile (general cholesterol, HDL cholesterol, LDL - cholesterol and triglycerides) between patients with DMT1 and DMT2 were found. Patients with DMT2 were found to have statistically higher BMI $\left(34,24 \pm 1,45\right.$ vs. $\left.26,38 \pm 1,64 \mathrm{~kg} / \mathrm{m}^{2}\right)$ and waist $(112,75 \pm 3,53$ vs. $100,76 \pm 5,51 \mathrm{~cm}$.) compared to patients with DMT1.

No differences in the total leukocyte count were found among the studied groups (Fig.1). Patients with DMT1 $\left(0,45 \pm 0,12 \times 10^{9} / I\right)$ and DMT2 $\left(0,45 \pm 0,15 \times 10^{9} / I\right)$ had a higher absolute monocyte count than the group of healthy individuals $\left(0,28 \pm 0,09 \times 10^{9} / \mathrm{l}\right)$ (Table 2$)$.

Results from flowcytometric analysis showed statistically higher absolute number of T-all lymph (CD3+), Th lymph (CD4+) and all NK (CD3\CD16+/ CD56), as well as a lower absolute number of Ts

Table 1. Clinical characteristics of patients with diabetes and the control group

\begin{tabular}{|c|c|c|c|c|}
\hline Index & DMT1 (22) & DMT2 (70) & Controls (21) & Significance $(p<0.05)$ \\
\hline Age (years) & $41,23 \pm 5,42$ & $56,58 \pm 2,25$ & $31,14 \pm 6,92$ & $\begin{array}{c}P<0,05 ; p^{*}<0,05 \\
p \neq<0,05\end{array}$ \\
\hline $\mathrm{BMI}\left(\mathrm{kg} / \mathrm{m}^{2}\right)$ & $26,38 \pm 1,64$ & $34,24 \pm 1,45$ & $24,32 \pm 2,71$ & $\begin{array}{c}P<0,05 ; p^{*}<0,05(0,04) \\
p \neq<0,05\end{array}$ \\
\hline Waist circumference $(\mathrm{cm})$ & $100,76 \pm 5,51$ & $112,75 \pm 3,53$ & $81,10 \pm 8,4$ & $\begin{array}{c}P<0,05(0,01) ; P^{*}<0,05 \\
p \neq<0,05\end{array}$ \\
\hline Systolic blood pressure ( $\mathrm{mm} \mathrm{Hg}$ ) & $129,77 \pm 9,59$ & $133,82 \pm 3,93$ & $116,67 \pm 15,52$ & $\begin{array}{c}P=0,35 ; p^{*}<0,05(0,03) \\
p \neq<0,05\end{array}$ \\
\hline Diastolic blood pressure $(\mathrm{mmHg})$ & $79,55 \pm 5,76$ & $82,87 \pm 2,08$ & $76,19 \pm 9,61$ & $\begin{array}{c}P=0,17 ; p^{*}=0,34 \\
p \neq<0,05(0,03)\end{array}$ \\
\hline $\mathrm{HbA} 1 \mathrm{c}(\%)$ & $8,29 \pm 1,0$ & $8,32 \pm 0,5$ & $4,71 \pm 0,31$ & $\begin{array}{c}P=0,95 ; p^{*}<0,05 \\
p \neq<0,05\end{array}$ \\
\hline Total cholesterol (mmol/l) & $5,25 \pm 0,56$ & $5,59 \pm 0,3$ & $4,52 \pm 0,5$ & $\begin{array}{c}P=0,27 ; p^{*}<0,05(0,01) \\
p \neq<0,05\end{array}$ \\
\hline HDL-cholesterol (mmol/l) & $1,33 \pm 0,18$ & $1,20 \pm 0,08$ & $1,26 \pm 0,24$ & $\begin{array}{c}P=0,19 ; p^{*}=0,54 \\
p \neq=0,51\end{array}$ \\
\hline LDL-cholesterol (mmol/l) & $3,05 \pm 0,42$ & $3,21 \pm 0,29$ & $2,58 \pm 0,62$ & $\begin{array}{c}P=0,57 ; p^{*}=0,06 \\
p \neq<0,05(0,03)\end{array}$ \\
\hline Triglycerides (mmol/l) & $2,46 \pm 1,24$ & $2,50 \pm 0,33$ & $1,06 \pm 0,33$ & $\begin{array}{c}P=0,91 ; p-<0,05(0,03) \\
p \neq<0,05\end{array}$ \\
\hline
\end{tabular}

Abbr.: P - DMT1 vs DMT2; $p^{*}-$ DMT1 vs controls; $p \neq$ DMT2 vs controls. 
(CD8+) and B (CD19+) lymph among all patients with DM compared to individuals from the control group. The Th/Ts ratio in patients with DM was also significantly higher in comparison with the control group and the referential values (from 1,1 to 1,5). No significant differences in the lymph subpopulations in the two investigated groups with DM were present (Table 2).

Among the patients with DMT2, a negative correlation was established between the total leukocyte count (from the automated counting), waist circumference $(r=-0,47 ; p=0,02)$, BMI $(r=-0,28 ; p=$ $0,02)$ and HOMA IR $(r=-0,47 ; p=0,02)$, as well as a positive correlation with $\mathrm{HOMA} \% \mathrm{~B}(r=0,5 ; p$ $=0,01)$. Glycemic control, estimated by $\mathrm{HBA} 1 \mathrm{c}$ showed a positive correlation with the total leukocyte count $(r=0,27 ; p=0,04)$ and NK cells $(r=$ $0,46 ; p=0,05)$. In the group of DMT2, a positive correlation between the levels of triglycerides and total leukocyte count $(r=0,36 ; p=0,003)$ and $T$ lymph [CD3+( $r=0,33 ; p=0,01) ; C D 4+(r=0,34$; $p=0,009)]$, as well as a negative correlation between the levels of HDL-cholesterol and total leukocyte count $(r=-0,33 ; p=0,005)$ were also found. Among the patients with DMT2, no association was found between the total and differential leukocyte count, lymph types and glycemic levels. A positive link between NK cells and BMI $(r=0,78 ; p=0,004)$ was established. Positive correlation between serum triglycerides and NK cells count $(r=0,6 ; p=$ 0,04 ) was found too.

\section{DISCUSSION}

Our results showed no differences in the total leukocyte and lymph count among patients with DMT1, DMT2 and healthy individuals. Similar results have been reported by Haider et al. for a

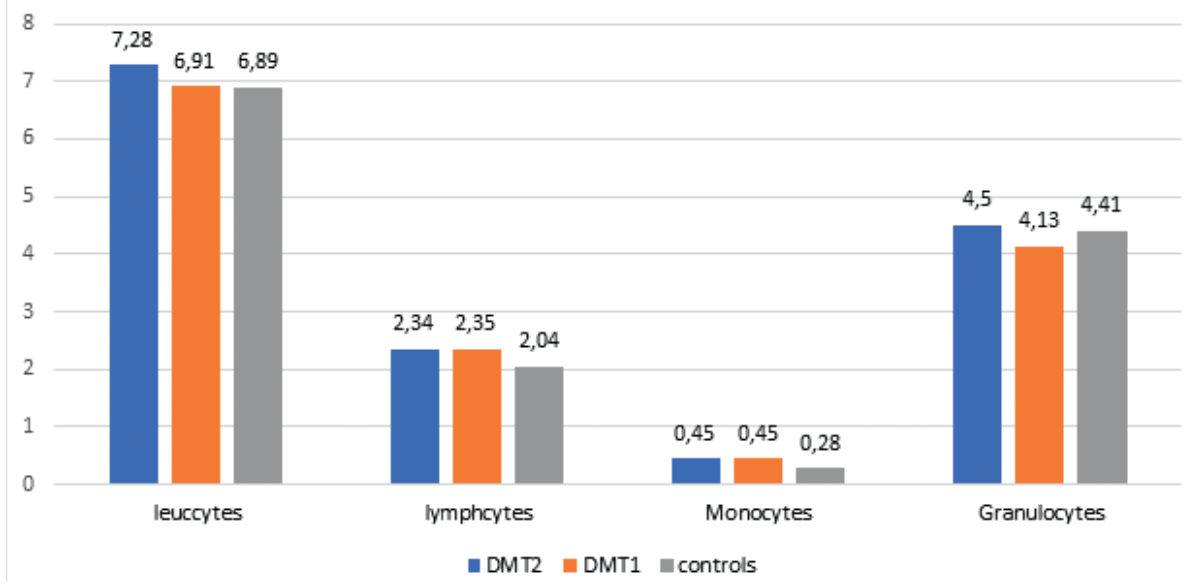

Fig. 1. Total and differential leucocytes count in diabetic patients (the results are present as absolute value $\times 10^{9} /$ )

Table 2. Absolute lymphocyte subpopulations count in diabetic patients

\begin{tabular}{|l|c|c|c|c|}
\hline & DMT1 $(n=22)$ & DMT2 $(n=70)$ & controls $(n=21)$ & significance $(p<0.05)$ \\
\hline All T lymphocytes (CD3+) & $1662.55 \pm 302.76$ & $1616.59 \pm 124.8$ & $1120.43 \pm 17.54$ & $\begin{array}{r}p N S(0,74) ; p^{*}<0,05 \\
p \neq<0,05\end{array}$ \\
\hline T-helpers (CD4+) & $1041.75 \pm 185.69$ & $1082.44 \pm 107.14$ & $728.81 \pm 14.43$ & $\begin{array}{r}p N S(0,7) ; p^{*}<0,05 \\
p \neq<0,05\end{array}$ \\
\hline T-suppress (CD8+) & $604.15 \pm 153.47$ & $515.10 \pm 67.25$ & $783.48 \pm 36.92$ & $\begin{array}{r}P N S(0,22) ; p^{*}<0,05 \\
p \neq<0,05\end{array}$ \\
\hline Th/Ts & $2.02 \pm 0.44$ & $2.36 \pm 0.37$ & $1.02 \pm 0.06$ & $\begin{array}{r}p N S(0,29) ; p^{*}<0,05 \\
p \neq<0,05\end{array}$ \\
\hline $\begin{array}{l}\text { B lymphocytes. } \\
\text { (CD19+) }\end{array}$ & $200.9 \pm 56.32$ & $186.02 \pm 26.07$ & $287.67 \pm 23.53$ & $\begin{array}{r}P N S(0,59) ; p^{*}<0,05 \\
p \neq<0,05\end{array}$ \\
\hline $\begin{array}{l}\text { NK cells } \\
\text { (CD31 CD16+/CD56) }\end{array}$ & $327.42 \pm 119.78$ & $385.0 \pm 86.82$ & $271.19 \pm 19.0$ & $\begin{array}{r}P N S(0,43) ; p^{*} N S(0,19) \\
p \neq<0,05\end{array}$ \\
\hline
\end{tabular}

Abbr.: P - DMT1 vs DMT2; $p^{*}-$ DMT1 vs controls; $p \neq$ DMT2 vs controls 
small group of 23 men with DMT 2 in Iraq [19]. A German Diabetes Study showed different results than ours, where a higher leukocyte and lymph count were observed among patients with DMT2 in comparison with DMT1 and healthy normoglycemic subjects. In this study, all patients (62 with DMT1 and 132 with DMT2) were with good metabolic control and a short duration of the diabetes $(1,29$ years for DMT1 and 1,98 years for DMT2). Higher levels of total cholesterol, LDL-cholesterol, triglycerides and lower levels of HDL-cholesterol were observed in patients with DMT2 [20]. The participants in our study with DM had poor glycemic control (HbA1c for DMT1-8,89\%; DMT2$8,32 \%)$ and a significantly longer duration of the diseases (DMT1-9,79 years; DMT2-6,12 years), without any differences in the lipid profile. Individuals with DM were found to have a higher absolute monocyte count compared to the healthy ones in the control group. These results can be explained with the fact that monocytes are antigen presenting cells which stimulate CD4+ T lymph to produce inflammatory cytokines such as TNF- $\alpha$ [21]. CD3+ lymph are the primary pool of specific immune cells. After the antigens stimulation, they differentiate to CD4+ and CD8+ T lymph- the main cellular modulators of the adaptive immune system. In the study carried out by us, the proportion and absolute count of non-specific T-lymph $(C D 3+)$ and Th (CD4+) were higher in comparison to those of healthy individuals, while Ts (CD8+) were lower. No statistical differences were found between DMT1 and DMT2. Our results match with those of the German Diabetes Study for patients with DMT2. Similar results were reported by researchers from Ukraine, which studied the effect of obesity and BMI on the lymph among DMT2 [22]. Both in innate and acquired immunity, NK cells produce a large number of cytokines such as TNF- $\alpha$, after activation. Among patients with DMT2, significantly higher levels of NK cells were found, compared to the healthy control group. Low levels of B-lymph in peripheral blood were also found. De Furiaa et al. established a promotive role of B-lymph for the development of DMT2 and their regulatory function in relation to T-lymph differentiation among those patients [23]. They have also reported a low absolute count of CD19+ lymph and a negative correlation with BMI among patients with IR and visceral obesity. Limiting factors exist in the design of our study, which may have affected the results. The prospective study carried out by us does not allow for the observed changes in cellular elements of innate and acquired immunity to be followed and evaluated. Secondly, phenotyping of immune cells was not possible after specific stimulation (specific anti-island antigens or functional stimuli). Cellular types were studied in peripheral blood, but without any possibility for their study in the pancreas or fat tissue.

In conclusion, our study demonstrates immune activation and dysregulation among the two types of diabetes. No quantity differences between DMT1 and DMT2 were found in lymph populations in peripheral blood. Aside from hyperglycemia in DMT2, a number of factors such as BMI, waist and lipid profile caused modulation of the elements of adaptive immunity.

Disclosure summary: The authors have nothing to disclose.

Acknowledgments: none.

\section{REFERENCES}

1. Damoiseaux JG, Tervaert JW. The definition of autoimmune disease: are Koch's postulates applicable? Neth J Med, 2002, 60:266-268.

2. Wållberg $M$, Cooke $A$. Immune mechanisms in type 1 diabetes. Trends Immunol, 2013, 34, 583-591.

3. Wentworth JM, Fourlanos S, Harrison LC. Reappraising the stereotypes of diabetes in the modern diabetogenic environment. Nat Rev Endocrinol, 2009, 5, 483-489.

4. KamradtT, MitchisonNA. Tolerance and autoimmunity. NEngl J Med, 2001, 344, 655-664.

5. Gallucci S, Matzinger P. Danger signals: SOS to the immune system. Curr Opin Immunol 2001, 13, 114-11.

6. Winer DA, Winer $S$, Shen L, et al. B cells promote insulin resistance through modulation of $T$ cells and production of pathogenic IgG antibodies. Nat Med, 2011, 17, 610-617.

7. Turner R, Stratton I, Horton V, et al. UK Prospective Diabetes Study Group. UKPDS 25: autoantibodies to islet-cell cytoplasm and glutamic acid decarboxylase for prediction of insulin requirement in type 2 diabetes. Lancet, 1997, 350, 1288-1293

8. Brooks-Worrell B, Narla R, Palmer JP. Islet autoimmunity in phenotypic type 2 diabetes patients. Diabetes Obes Metab, 2013, 15(Suppl. 3), 137-140.

9. Feuerer M, Herrero L, Cipolletta D, et al. Lean, but not obese, fat is enriched for a unique population of regulatory $T$ cells that affect metabolic parameters. Nat Med, 2009,15, 930-939.

10. Strissel KJ, DeFuria J, Shaul ME et al. T-cell recruitment and Th1 polarization in adipose tissue during diet-induced obesity in C57BL/6 mice. Obesity, 2010,18, 1918-1925. 
11. Fabbrini E, Cella M, McCartney SA, et al. Association between specific adipose tissue CD4+ T-cell populations and insulin resistance in obese individuals. Gastroenterology, 2013, 145, 366-374.

12. McLaughlin T, Liu LF, Lamendola $C$, et al. T-cell profile in adipose tissue is associated with insulin resistance and systemic inflammation in humans. Arterioscler Thromb Vasc Biol, 2014,34, 2637-2643.

13. Butcher MJ, Hallinger D, Garcia E, et al. Association of proinflammatory cytokines and islet resident leucocytes with islet dysfunction in type 2 diabetes. Diabetologia, 2014, 57, 491-501.

14. Donath MY, Shoelson SE. Type 2 diabetes as an inflammatory disease. Nat Rev Immunol, 2011,11, 98-107.

15. Wilkin TJ. The accelerator hypothesis: weight gain as the missing link between type 1 and type 2 diabetes. Diabetologia, 2001, 44, 914-922

16. Tuomi T, Carlsson A, $\mathrm{Li} \mathrm{H}$ et al. Clinical and genetic characteristics of type 2 diabetes with and without GAD antibodies. Diabetesq 1999, 48, 150-7.

17. Diagnosis and Classification of Diabetes Mellitus. Diabetes, Care, 2011, 34(Suppl 1), 62-69.

18. Thomas G. Pickering, MD et al. Recomendation for blood pressure measurment in humans and experimental animals, Hypertension, 2004, 45, 142.
19. Haider K. Zaidan, Israa H. Ibrahim, Ali H. Al Saadi, Mufeed J.Ewadh, Qasim M. Ali Al Ameri Total and differential leukocytes count in type 2 diabetes mellitus patients in Iraq. Research in Biotechnology, 2012, 3(2)1, 32-40.

20. Menart- Houtermans B., Rütter R, Nowotny B et al. Leukocyte Profiles Differ Between Type 1 and Type 2 Diabetes and Are Associated With Metabolic Phenotypes: Results From the German Diabetes Study (GDS). Diabetes Care, 2014, 37(8), 2326-2333.

21. Bradshaw EM, Raddassi K, Elyaman W. et al. Monocytes from patients with type 1 diabetes spontaneously secrete proinflammatory cytokines inducing Th17 cells. Journal of immunology, 2009, 183, 4432-9.

22. Zak K.P., Mankovsky B.M., Kondratska I.N et al. Afanasyeva Immunity in patients with type 2 diabetes mellitus with concomitant metabolic syndrome/ obesity. Communication 1. Composition of blood leukocytes, immunophenotype of lymphocytes, and ultrastructure of neutrophils. Endokrynologia, 2013, 18 (1), 27-36.

23. Jason De Furiaa, Anna C. Belkinab, Madhumita Jagannathan-Bogdanc et al. B Cells promote inflammation in obesity and type 2 diabetes through regulation of T-cell function and an inflammatory cytokine profile. PANAS, 2010, 110, 51335138. 